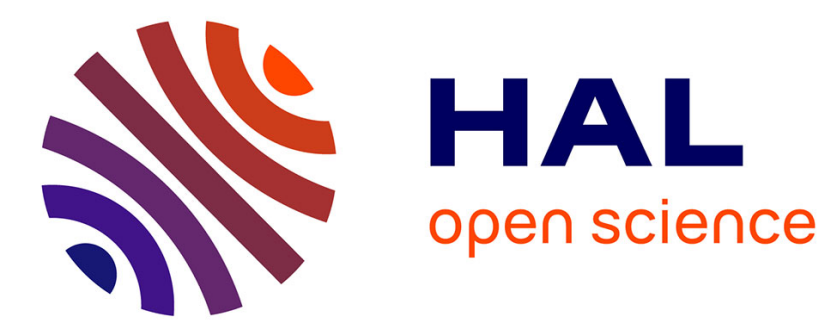

\title{
Asymmetric axonal edge guidance: a new paradigm for building oriented neuronal networks
}

Renaud Renault, Jean-Baptiste Durand, Jean-Louis Viovy, Catherine Villard

\section{To cite this version:}

Renaud Renault, Jean-Baptiste Durand, Jean-Louis Viovy, Catherine Villard. Asymmetric axonal edge guidance: a new paradigm for building oriented neuronal networks. Lab on a Chip, 2016, 16 (12), pp.2188-2191. 10.1039/C6LC00479B . hal-01336980

\section{HAL Id: hal-01336980 \\ https://hal.sorbonne-universite.fr/hal-01336980}

Submitted on 24 Jun 2016

HAL is a multi-disciplinary open access archive for the deposit and dissemination of scientific research documents, whether they are published or not. The documents may come from teaching and research institutions in France or abroad, or from public or private research centers.
L'archive ouverte pluridisciplinaire HAL, est destinée au dépôt et à la diffusion de documents scientifiques de niveau recherche, publiés ou non, émanant des établissements d'enseignement et de recherche français ou étrangers, des laboratoires publics ou privés. 


\title{
Asymmetric axonal edge guidance : a new paradigm for building oriented neuronal networks ${ }^{\dagger}$
}

\author{
Renaud Renault, ${ }^{\text {abc }}$ Jean-Baptiste Durand, ${ }^{\text {abc }}$ Jean-Louis Viovy ${ }^{\text {abc }}$ and Catherine Villard ${ }^{* a b c}$
}

\begin{abstract}
We present a novel kind of directional axon guides for brain-on-a-chip applications. Contrarily to previous works, the directionality in our design is created by rerouting axons growing in the unwanted direction back to their original compartment while leaving the other growth direction unaffected. This design yields state-of-the-art levels of directionality without the disadvantages of previously reported technologies.
\end{abstract}

In vitro reconstruction of neural circuits represents an interesting alternative to animal models or brain slices, and microfluidics has emerged as an important technology to that end. The connectivity between neurons in microfluidic chips is usually controlled using microchannels that physically allow axons to grow and connect different populations of neurons while keeping the somas and dendrites in their respective compartments. ${ }^{1}$ In numerous cases, it is desirable to have unidirectional connections between neuronal populations, for instance in order to recreate specific neural pathways involved in neurodegenerative diseases ${ }^{2}$ or to create functional neuronal devices. ${ }^{3}$ Several active methods using light, ${ }^{4}$ chemical gradients, ${ }^{5}$ or dielectrophoretic forces ${ }^{6}$ exist. However, these techniques are costly in terms of manufacturing time and instrumentation, and require synchronization of stimuli with axonal development, making experiment parallelization difficult. An alternative consists in using only geometrical constraints to passively control the direction of axonal growth. ${ }^{7,8}$

So far, the most characterized and efficient design consists in tapered microchannels, acting like axon diodes. It uses the differential entrance probability of axons at either the wide or the narrow opening of the channels to promote unidirectional connectivity. ${ }^{7}$ Yet, the efficiency of this design critically depends on the width of the narrow side which cannot be reduced below a critical size without important drawbacks, such as difficult and irreproducible micro-fabrication, limited axonal transmission in the desired direction leading to a lower overall connectivity, restricted access of diffusive factors through the channels and mechanical constriction, compromising long term axonal integrity.

We propose in this paper a completely new axon diode design which yields state-of-the-art direction selectivity without the inherent drawbacks of tapered channels. Our approach stems from the observation that (1) cortical axons tend to grow along the edges (dihedral junctions between horizontal and vertical walls) of 3D guiding microstructures, and that (2) they escape this guidance if the edge's direction changes too abruptly, due to their non-zero bending stiffnesses. ${ }^{9,10}$ In our design, we implemented a selective "return to sender" strategy by exploiting this phenomenon, redirecting to their original compartment axons growing in the unwanted "reverse" direction via U-shaped lateral connections. We quantitatively evaluated the efficiencies of different variations of this basic concept regarding their direction selectivity, and demonstrate that they constitute a major improvement over the tapered channels already used by the brain-on-a-chip community. $2,7,11,12$

Over the course of different experiments using PDMS microchips, we observed that cortical axons tend to follow the edges of microstructures in a very consistent manner. In order to explore this phenomenon in more details, PDMS sectors (radius $30 \mu m$ ) with various angles (Figure $1 \mathrm{~A}$ and $\mathrm{B}$ ) were used to determine the preferred trajectories of cortical axons (see experimental section ${ }^{\dagger}$ ) initially growing along the edges of microstructures when they meet an abrupt change of direction $\alpha$. Our results show that edge guidance predominates when $\alpha \leq 26^{\circ}$. In contrast, straight axonal trajectory prevails over edge guidance when $\alpha \geq 84^{\circ}$. These observations were used to control the directionality of axonal flow. Our design is based on straight channels with a uniform width $(10 \mu \mathrm{m})$ and height $(5 \mu \mathrm{m})$ connecting two chambers, similarly to the original microchannels. ${ }^{1}$ However, in this new design, the channels are interconnected by curved microchannels (arches). When arriving at a bifurcation, axons following the edges of the channels in the reverse direction experience a smooth change of orientation and are expected to keep following the edges inside the arches to be progressively diverted into the adjacent channel and make U-turns towards their original chamber (Figure $1 \mathrm{C}$, red axon). Conversely, axons emitted in the forward direction experience instead an acute cusp. In this case, axons are expected to be released from the edges and resume their trajectories in the same straight microchannel after the junction (green axon).

A clear demonstration of these two behaviors are shown in Figure 2 and Movie S1 $1^{\dagger}$. Notice that the positions of the arches were shifted relatively to one another to firmly redirect the axons in the main channels. In designs involving unshifted arches, some axons achieved lateral escape along juxtaposed arches (see Movie S2 ${ }^{\dagger}$ ).

Institut Curie, PSL Research University, CNRS, UMR 168 Physico-Chimie Curie, 75005, Paris, France.

Sorbonne Universités, UPMC Univ, 75006, Paris, France.

Institut Pierre-Gilles de Gennes pour la microfluidique, 75005, Paris, France.

*E-mail: catherine.villard@curie.fr

† Electronic Supplementary Information (ESI) available: [details of any supplementary information available should be included here]. See DOI: 10.1039/b000000x/ 


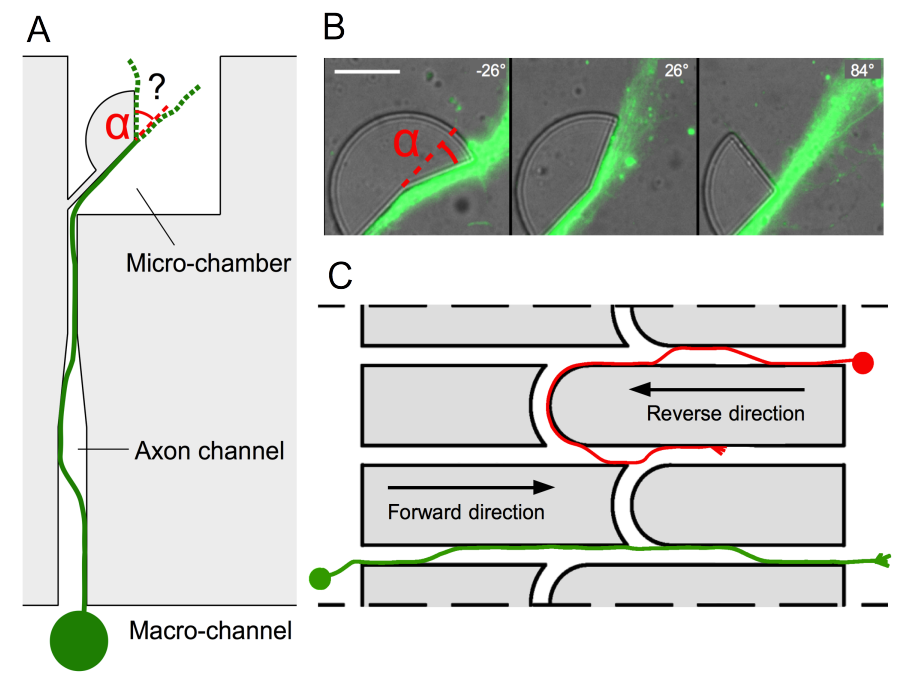

Fig. 1 Edge guidance and critical release angle. A) Typical design of the structures used to determine the value of the critical release angle, consisting in a $5 \mu \mathrm{m}$ high PDMS microchamber containing an incomplete cylinder with a $30 \mu \mathrm{m}$ radius, and forming sectors of various angles. The change in edge direction, $\alpha$, is defined as positive for convex corners. Axons (in green) are initially directed to the first edge of the sector through a tapered microchannel, initiating edge guidance. When they reach the cusp, they can either continue straight ahead or bend to keep following the edge of the structure, depending on the value of $\alpha$. The two possible trajectories are represented with dashed lines. B) Superimposition of DIC images showing a series of obstacles with different $\alpha$ values (indicated in white) with fluorescent images of cortical axons (green, microtubules) after 10 Days In Vitro (DIV). C) Schematic showing our design, which uses a combination of straight and curved microchannels (arches). The expected trajectories of axons in the forward (green) and reverse (red) directions are indicated.

In order to assess quantitatively the filtering capabilities of our designs, and given the difficulty to count tightly packed axons growing inside microstructures, we used fluorescent neurons (extracted from tdTomato mice, details of the experimental procedures and ethical approval are provided in the ESI ${ }^{\dagger}$ ) to readily estimate the number of axons in different areas, assumed to be proportional to the associated fluorescence intensities. Although this particular staining is not specific for axons, previous studies have shown that dendrites are found only in the proximal part of guiding structures and that only axons remain past a few tens of microns, ${ }^{13}$ leaving very little doubt on the axonal nature of the neurites present in the terminal segments of our microchannels. Axonal transmission used in our quantification method was calculated for each microchannel by normalizing the fluorescence intensity in the terminal segment to the fluorescence in the associated somatodendritic compartments (see experimental section ${ }^{\dagger}$ ). The direction selectivity coefficient for each design was defined as the ratio between the average forward and reverse axonal transmission. Note that in order to minimize the variability in soma densities and other hidden variables that could distort the comparison between forward and reverse transmission, we designed each PDMS chip to present both orientations to a shared neuronal compartment.

We firstly evaluated the filtering capabilities of different variations of our design in a simple case study where only one side was seeded, similarly to the protocols used for previous designs characterization. ${ }^{7}$. The direction selectivity was evaluated for designs with one, two and three stages of arches, as well as a variation of the three stages geometry where, in addition to the arches inside the microchannels, the edges of the neuronal compartments between the microchannels openings were arched as well. This particular design will be referred as the " 3 arches + " pattern in the remainder of the article. Figure 3 presents the results obtained for the different designs, both as a graph (top) presenting the whole distribution of axonal transmission in the forward (green) and reverse (red) directions, and a visual representation (bottom) displaying an average intensity map of axonal density.

We observed a clear reduction of axonal transmission in the reverse direction as the number of arches increased, down to almost undetectable levels in the particular cases of 3 arches and 3 arches + patterns. In agreement with this observation, the axonal intensity inside the microchannels decreased by steps at each layer of arches, expressing the fact that more and more axons are filtered at each stage. Although forward transmission was slightly decreased by the introduction of additional layers of arches, the selectivity, most importantly, kept increasing $(1.85 \pm 0.33,11.84 \pm 2.45$ and $13.86 \pm 3.00$ for the 1,2 and 3 arches geometries, respectively). Strikingly, forward axonal transmission significantly increased ( $\mathrm{p}>0.0001$ ) in the 3 arches + patterns in comparison to the 3 arches patterns, while axonal transmission in the reverse direction remained low. Our explanation is that the curved walls in contact with the emitting population act as funnels, guiding axons towards the entrance of the microchannels, while they act as deflectors in the reverse direction. This configuration, combining improved axonal collection on the forward entry side and axonal diversion on the opposite side, consequently reaches the striking directional selectivity of $21.67 \pm 3.19$. In regard to previous technologies, the 3 arches + design gives state of the art performances, with less than 5\% reverse growth and without the drawbacks of tapered diodes, or geometrical constrictions in general. 7,8 


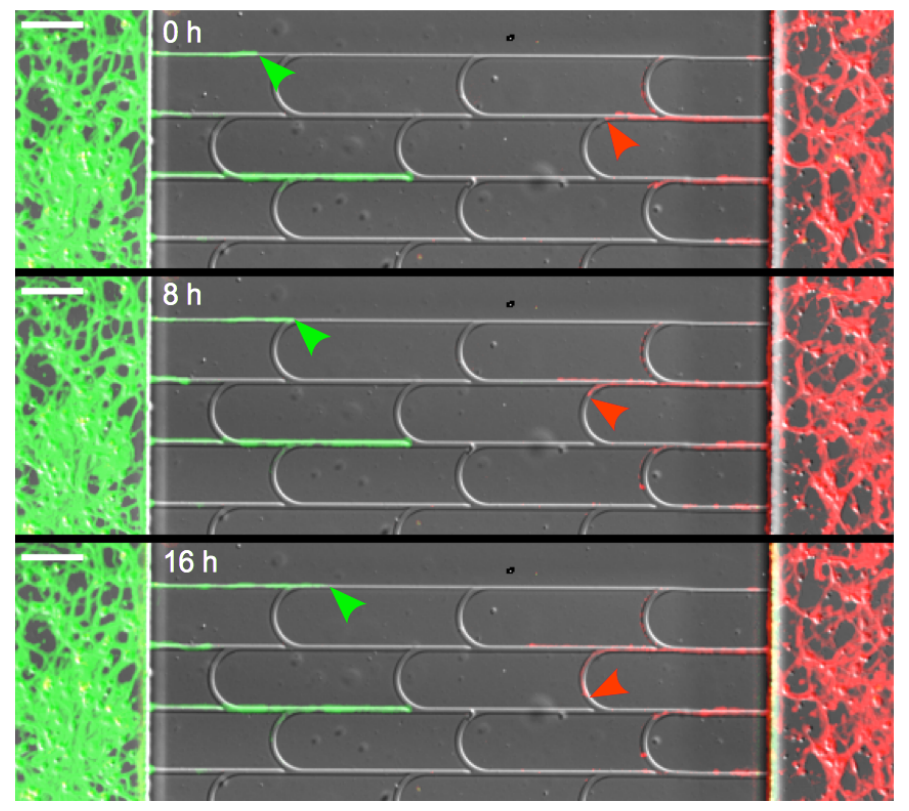

Fig. 2 Selective return-to-sender. Superimposed DIC and fluorescence images of a chip seeded with two different neuronal populations (expressing tdTomato or GFP-LifeAct). Green axons, growing in the forward direction, mostly ignore the junctions (green arrowheads), while red axons are rerouted (red arrowheads) back to their original compartment. The first frame (0h) was taken 40 hours after seeding. Scale bar : $100 \mu m$

We then measured the performances of our microchannels designs in connecting directionally two neuronal populations by seeding non fluorescent neurons in the second chamber. As in the former case, Figure 4 provides a plot of axonal transmission distributions as well as a visual representation of the filtering effect of arches. Similarly to the one-side-seeded case, the selectivity increased with the number of arches, the 3 arches + configuration yielding the highest selectivity (10.02 \pm 1.26$)$. The major difference with the one-population-case was the increase of axonal transmission in the reverse direction, which impaired the filtering power of arches. The intensity profiles of Figure 5 show indeed that the fraction of axons making U-turns after each layer is reduced from about $63 \%$ (A) when one side is seeded, to about $47 \%$ (B) when both sides are seeded.

This reduction in selectivity can be explained by the interfering guidance cues provided by axons growing in the opposite direction. In line with this, note how stalling and/or branching of axons (illustrated in Figure S3 ${ }^{\dagger}$ ), revealed by the peaks in the density profiles of Figure 5, are less pronounced when both sides are seeded. Since stalling and branching are due to the lack of guiding cues, ${ }^{14}$ the reduction of the peaks matches well with the hypothesis that axons growing in the opposite direction provide additional guiding cues. In spite of this phenomenon, a selectivity of $90 \%$ was attainable, and it could be increased further simply by using additional layers of arches.

\section{Conclusions}

We showed that unidirectional axonal projections between neuronal populations grown inside microfluidic chips can be obtained using a novel microchannel geometry, which exploits edge guidance to operate an efficient and selective rerouting of axons growing in the unwanted direction. In addition, the elementary units (arches) can easily be stacked together in order to create tailored levels of directionality. This strategy does not necessitate the use of extremely narrow ${ }^{7}$ or innerly structured ${ }^{8}$ microchannels. Consequently, our approach offers an easier microfabrication, a higher axonal transmission, and a better diffusion of possibly important molecules in the axon channels. Besides the important implications of these axon diodes for the reconstruction of oriented neural pathways, the original concepts of edge guidance and critical release angle could be developed into many other practical applications and benefit the growing brain-on-a-chip community far beyond the proof of concept provided by this particular study.

\section{Acknowledgements}

We thank Daniela Vijgnevijc from the Curie Institute for providing us with the tdTomato mice, Pablo Vargas and Ana-Maria Lennon for providing us with the GFP LifeAct mice, Isabelle Grandjean and Manon Chartier from the animal facility of the Curie Institute for their crucial support for mice. This work was performed in part in the microfabrication cleanroom of the Institut Pierre-Gilles de Gennes. This work was supported by ERA-Net NEURON (MICRODEG), the Neuroscreen ANR project, European research Council Advanced Grant $N^{\circ}: 321107$ "CellO", ANR Investissement d'avenir, and the IPGG Labex and Equipex. 


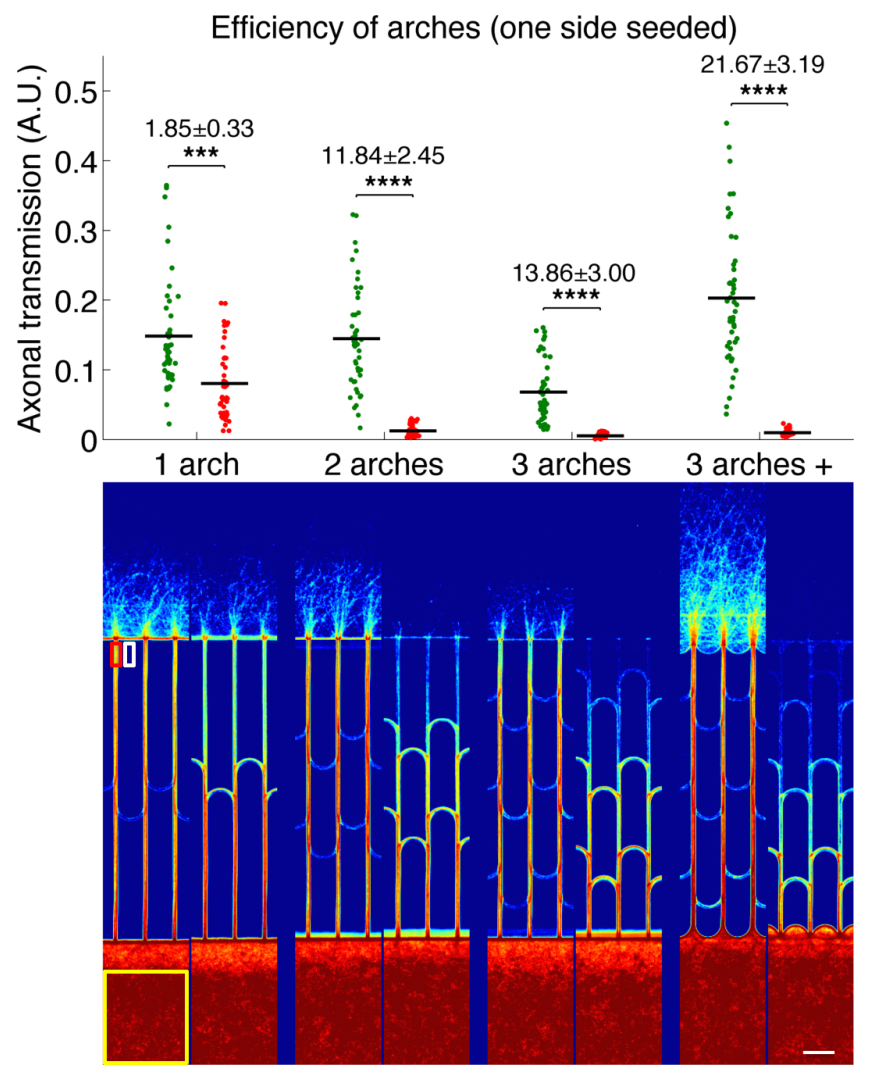

Fig. 3 Intrinsic direction selectivity of arches, one side seeded. Top: distribution of axonal transmission for the different designs both in the forward (green) and reverse (red) directions. The mean is indicated as a horizontal black segment for each distribution. The selectivity ratios are indicated, as well as the statistical significance of the observed directionality $\left({ }^{*}: P<0.05,{ }^{* *}: P<0.01,{ }^{* * *}: P<0.0001\right.$ and ${ }^{* * * *}: P<0.000001$, two-tailed Student's t-test for independent samples) Bottom: average intensity maps of fluorescent (tdTomato) axons in the different designs (log scale). The ROI from which are extracted the initial neuronal density, terminal axonal density and background allowing to calculate axonal transmission are represented for one channel with yellow $(\mathrm{Y})$, red $(\mathrm{R})$ and white $(\mathrm{W})$ rectangles respectively $\left(\right.$ Axonal transmission $\left.=\frac{R-W}{Y}\right)$. Experiment realized after 8 DIV. Scale-bar $100 \mu \mathrm{m}$.

\section{References}

1 J. W. Park, B. Vahidi, A. M. Taylor, S. W. Rhee and N. L. Jeon, Nat Protoc, 2006, 1, 2128-2136.

2 B. Deleglise, S. Magnifico, E. Duplus, P. Vaur, V. Soubeyre, M. Belle, M. Vignes, J.-L. Viovy, E. Jacotot, J.-M. Peyrin and B. Brugg, Acta Neuropathol Commun, 2014, 2, 145 .

3 O. Feinerman, A. Rotem and E. Moses, Nat Phys, 2008, 4, 967-973.

4 A. Ehrlicher, T. Betz, B. Stuhrmann, D. Koch, V. Milner, M. G. Raizen and J. Kas, Proc Natl Acad Sci U S A, 2002, 99, 16024-16028.

5 I. Dupin, L. Lokmane, M. Dahan, S. Garel and V. Studer, Neural Dev, 2015, 10, 5.

6 T. Honegger, M. A. Scott, M. F. Yanik and J. Voldman, Lab Chip, 2013, 13, 589-598.

7 J.-M. Peyrin, B. Deleglise, L. Saias, M. Vignes, P. Gougis, S. Magnifico, S. Betuing, M. Pietri, J. Caboche, P. Vanhoutte, J.-L. Viovy and B. Brugg, Lab Chip, 2011, 11, 3663-3673.

8 J. le Feber, W. Postma, E. de Weerd, M. Weusthof and W. L. C. Rutten, Front Neurosci, 2015, 9, 412.

9 R. M. Smeal, R. Rabbitt, R. Biran and P. A. Tresco, Ann Biomed Eng, 2005, 33, 376-382.

10 S. Roth, G. Bugnicourt, M. Bisbal, S. Gory-Faure, J. Brocard and C. Villard, Small, 2012, 8, 671-675.

11 J. W. Park, H. J. Kim, M. W. Kang and N. L. Jeon, Lab Chip, 2013, 13, 509-521.

12 R. Renault, N. Sukenik, S. Descroix, L. Malaquin, J.-L. Viovy, J.-M. Peyrin, S. Bottani, P. Monceau, E. Moses and M. Vignes, PLoS One, 2015, 10, e0120680.

13 A. Yamada, M. Vignes, C. Bureau, A. Mamane, B. Venzac, S. Descroix, J.-L. Viovy, C. Villard, J.-M. Peyrin and L. Malaquin, Lab Chip, 2016, -.

14 G. Gallo, Dev Neurobiol, 2011, 71, 201-220. 


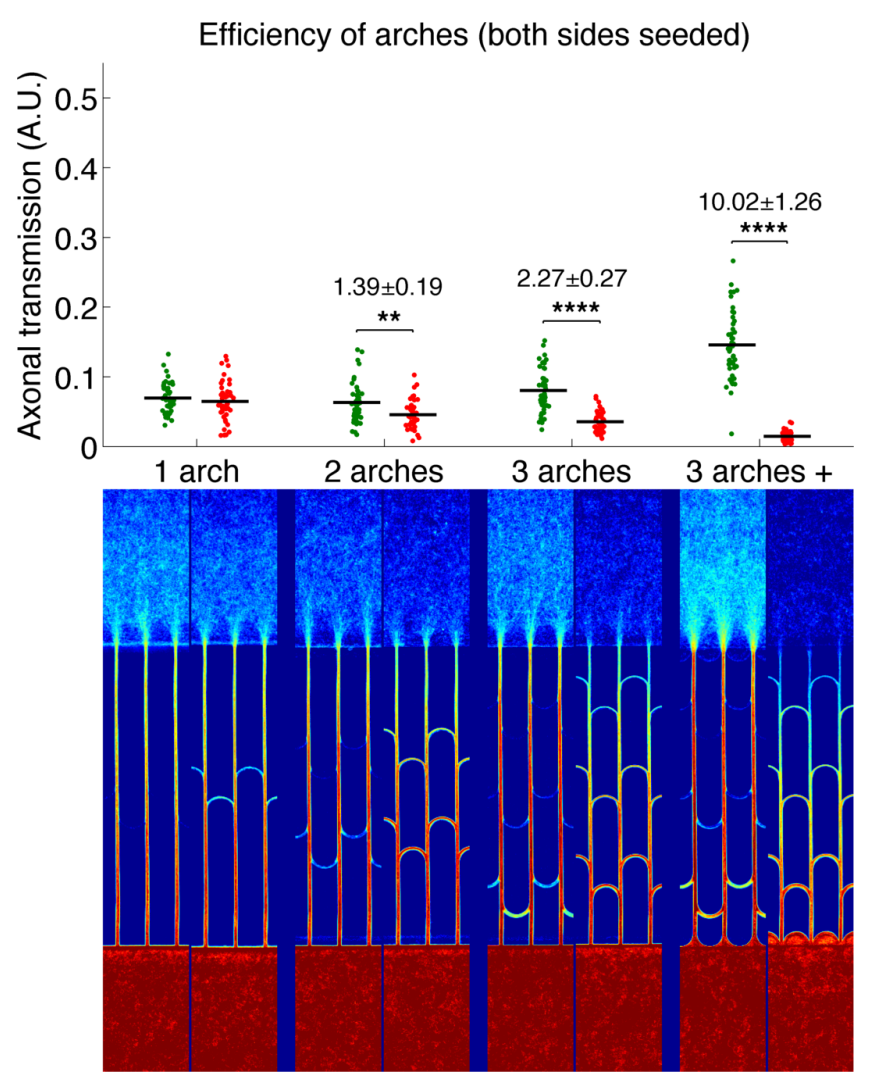

Fig. 4 Direction selectivity of arches, both sides seeded. Top: distribution of axonal transmission for the different designs both in the forward (green) and reverse (red) directions. The mean is indicated as a horizontal black segment for each distribution. The selectivity ratios are indicated, as well as the statistical significance of the observed directionality $\left(^{*}: \mathrm{P}<0.05,{ }^{* *}: \mathrm{P}<0.01,{ }^{* * *}: \mathrm{P}<0.0001\right.$ and ${ }^{* * * *}: \mathrm{P}<0.000001$, two-tailed Student's t-test for independent samples) Bottom: average intensity maps of axons in the different designs (log scale). Note that the top chambers are seeded with non-fluorescent neuronsExperiment realized after 8 DIV.

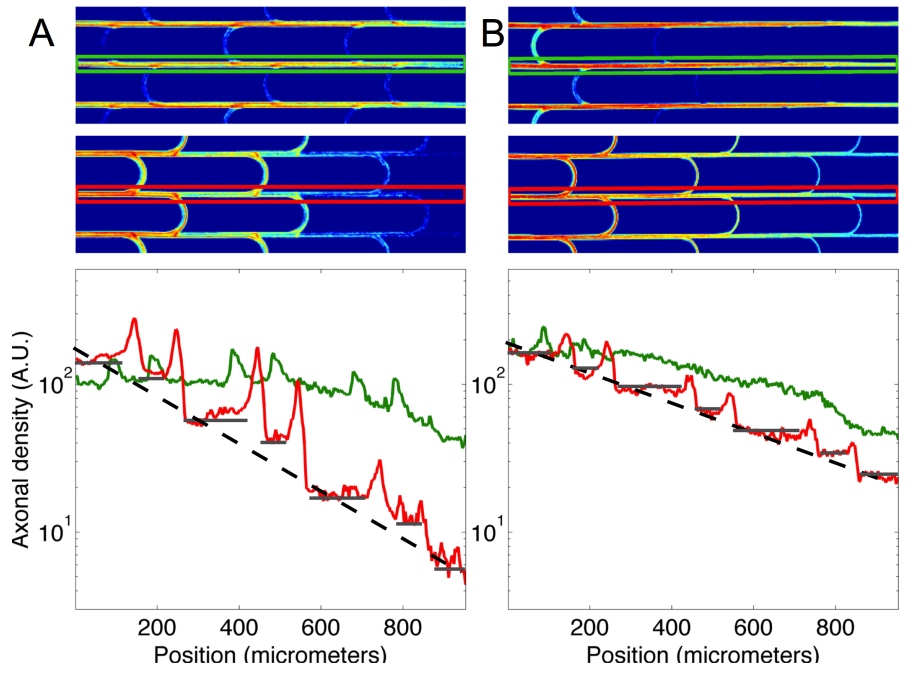

Fig. 5 Axonal density profiles along microchannels. A) Intensity profiles for the forward (greens) and reverse (red) directions are displayed for the 3 arches design. The ROls from which the profiles were computed are displayed with matching colors on the top pictures. The dashed line is a visual aid indicating the geometric progression of cumulated filtering. The contribution of each arch in the stepwise reduction of axonal transmission is underlined using dark gray segments. B) Same as A) in the both-sides-seeded case. 